\section{REFERENCES}

1. Parkes SL, Ravassard PM, Cerpa JC, Wolff M, Ferreira G, Coutureau E. Insular and ventrolateral orbitofrontal cortices differentially contribute to goal-directed behavior in rodents. Cereb Cortex. 2018;28:2313-25.

2. Whyte AJ, Kietzman HW, Swanson AM, Butkovich LM, Barbee BR, Bassell GJ, et al. Reward-related expectations trigger dendritic spine plasticity in the mouse ventrolateral orbitofrontal cortex. J Neurosci. 2019;39:4595-605.

3. Namboodiri VMK, Otis JM, van Heeswijk K, Voets ES, Alghorazi RA, RodriguezRomaguera J, et al. Single-cell activity tracking reveals that orbitofrontal neurons acquire and maintain a long-term memory to guide behavioral adaptation. Nat Neurosci. 2019;22:1110-21.
4. Groman SM, Keistler C, Keip AJ, Hammarlund E, DiLeone RJ, Pittenger C, et al. Orbitofrontal circuits control multiple reinforcement-learning processes. Neuron. 2019. https://doi.org/10.1016/j.neuron.2019.05.042.

5. Schoenbaum G, Setlow B, Saddoris MP, Gallagher M. Encoding predicted outcome and acquired value in orbitofrontal cortex during cue sampling depends upon input from basolateral amygdala. Neuron. 2003;39:855-67.

6. Chau BK, Sallet J, Papageorgiou GK, Noonan MP, Bell AH, Walton ME, et al. Contrasting roles for orbitofrontal cortex and amygdala in credit assignment and learning in macaques. Neuron. 2015;87:1106-18.

\title{
Regulation of fear extinction and relapse by hippocampal
}

\section{engrams}

\author{
Michael R. Drew iD ${ }^{1}$ and Emma T. Brockway ${ }^{1}$ \\ Neuropsychopharmacology (2020) 45:228-229; https://doi.org/10.1038/s41386-019-0481-x
}

Maladaptive fears are often treated using therapies based on extinction-re-exposure to a feared stimulus in a safe environment. Although these treatments can be effective, relapse is common [1]. Since the time of Pavlov, relapse has been thought to indicate that extinction is new learning, not unlearning [2]. From this perspective, relapse is a mnemonic phenomenon in which two opposing memory traces vie for expression. Recent work suggests that the hippocampus is an arena in which this competition plays out.

Experiments in mice demonstrate that the hippocampal dentate gyrus (DG) generates a contextual fear "engram." Immediate-early gene-based tagging of neurons active during acquisition of contextual fear-fear of the chamber in which a footshock was given-shows that these neurons are reactivated during recall of contextual fear [3]. Furthermore, optogenetic experiments demonstrate that this reactivation is necessary and sufficient for expression of contextual fear [3, 4]. Two recent studies investigated what happens to hippocampal fear ensembles when contextual fear is extinguished.

The first of these studies, by Lacagnina et al. [5], used a transgenic mouse line to tag neurons active during acquisition or extinction of contextual fear. During a test session shortly after extinction training, fear acquisition neurons were suppressed and extinction neurons were reactivated. A month after extinction training mice displayed spontaneous recovery (relapse) of fear, and the pattern reversed: fear acquisition neurons were reactivated while extinction neurons were suppressed. The results suggest that the DG generates distinct fear acquisition and extinction representations, and competition between these representations determines whether fear is suppressed or recovers after extinction. Consistent with this interpretation, optogenetic manipulations demonstrated that reactivation of extinction neurons is necessary for suppression of fear after extinction, whereas reactivation of fear acquisition neurons is necessary for spontaneous recovery [5]

The other recent study, by Khalaf et al. [6], highlights that fear acquisition neurons also play an important role in extinction learning. In this study, neurons active during recall of a remote fear memory (acquired a month before extinction training) were tagged. Reactivation of these neurons was necessary for effective extinction, and artificial stimulation of these neurons improved extinction. For extinction to be effective, the fear acquisition memory must be reactivated during extinction training. Whether these findings apply to recent fear memories, like those studied in the Lacagnina et al. [5] experiments, is not yet known.

These studies reveal new ensemble mechanisms of fear extinction and relapse and raise some interesting questions. For instance, what aspects of extinction training stimulate creation of hippocampal extinction representations, and how do these representations suppress fear? Do they do so by activating extra-hippocampal fear-suppressive networks? In the Lacagnina study, why did fear acquisition neurons become more active over time and extinction neurons less active? Is it because of intrinsic differences between the two populations, changes in upstream input pathways, or different plasticity mechanisms involved in fear acquisition and extinction? Addressing these and other questions raised by the Lacagnina and Khalaf papers may provide keys to making extinction more resistant to relapse.

\section{FUNDING AND DISCLOSURE}

M.R.D. was supported by $\mathrm{NIH}$ grants R01 MH102595 and R01 $\mathrm{MH} 117426$. E.T.B. was supported by NIH grant T32MH106454. The authors declare no competing interests.

\section{ADDITIONAL INFORMATION}

Publisher's note: Springer Nature remains neutral with regard to jurisdictional claims in published maps and institutional affiliations.

\section{REFERENCES}

1. Vervliet B, Craske MG, Hermans D. Fear extinction and relapse: state of the art. Annu Rev Clin Psychol. 2013;9:215-48. 
2. Bouton ME, Westbrook RF, Corcoran KA, Maren S. Contextual and temporal modulation of extinction: behavioral and biological mechanisms. Biol Psychiatry. 2006;60:352-60.

3. Denny CA, Kheirbek MA, Alba EL, Tanaka KF, Brachman RA, Laughman KB, et al. Hippocampal memory traces are differentially modulated by experience, time, and adult neurogenesis. Neuron. 2014;83:189-201.

4. Liu X, Ramirez S, Pang PT, Puryear CB, Govindarajan A, Deisseroth K, et al. Optogenetic stimulation of a hippocampal engram activates fear memory recall. Nature. 2012;484:381-385.
5. Lacagnina AF, Brockway ET, Crovetti CR, Shue F, McCarty MJ, Sattler KP, et al. Distinct hippocampal engrams control extinction and relapse of fear memory. Nat Neurosci. 2019;22:753-61.

6. Khalaf O, Resch S, Dixsaut L, Gorden V, Glauser L, Gräff J. Reactivation of recallinduced neurons contributes to remote fear memory attenuation. Science. 2018;360:1239-42.

\title{
The complexity of pharmacology of cannabidiol (CBD) and its implications in the treatment of brain disorders
}

\author{
Sonja Elsaid ${ }^{1,2}$ and Bernard Le Foll ${ }^{1,2,3}$ \\ Neuropsychopharmacology (2020) 45:229-230; https://doi.org/10.1038/s41386-019-0518-1
}

Cannabidiol (CBD) is one of the major cannabinoid constituents of the Cannabis plant. Recently, CBD has sparked the interest of medical researchers because of its more than 65 identified molecular targets. Of those, mostly studied in brain disorders are cannabinoid, $5 \mathrm{HT}_{1 \mathrm{~A}}$ receptors, G-protein receptor protein 55 (GPR55), transient receptor potential (TRP) channels, and cytochrome P450s [1]. Here we discuss possible mechanisms of actions of $C B D$ in several brain disorders.

The evidence suggests that the antiepileptic potential of CBD may be via its modulation of TRP (vanilloid 1 and TRPA), potassium channels, NMDA receptors, and more recently by the interaction with GPR55 to reduce neuronal excitability [1, 2]. Although in the US, CBD is currently prescribed as an adjuvant treatment for seizures in Lennox-Gastaut and Dravet syndromes, as well as tuberous sclerosis complex, it is still unknown if CBD's antiepileptic properties are due to its direct interaction with the molecular targets, or possibly through potentiating effects of antiepileptic treatments by modulation of cytochrome P450s [1, 3].

CBD exhibited anxiolytic properties by acting on the $5 \mathrm{HT}_{1 \mathrm{~A}}$ receptors in animal models [1]. Most recently, an in vitro study showed that CBD might also elicit anxiolytic effects by allosterically modulating $\mathrm{GABA}_{\mathrm{A}}$ receptors [4]. Human studies using $\mathrm{CBD}$ were limited to assessing the short-term effects of $C B D$ on social anxiety disorder (SAD) [1].

By the mechanism of action on the $C B 1$ receptor, $C B D$ attenuated behavioral responses to different forms of aversive memories in rodent PTSD models [5]. Although in human studies, CBD was associated with reduced PTSD symptomatology, the evidence is only limited to case studies, while possibly being confounded by the co-administration of other psychiatric treatments [6].

The antidepressant properties of $\mathrm{CBD}$ by activation of $5 \mathrm{HT}_{1 \mathrm{~A}}$ receptors were revealed in animal models of depression [1]. However, to date, CBD's effects on clinical depression have not been studied.
CBD has been proposed to have anti-psychotic effects by modulating dopamine D2, cannabinoid receptors, and TRPV1 channels; however, these mechanisms are somewhat speculative, given the lack of reproducibility of findings. In human studies, CBD produced conflicting evidence to either augment or improve the symptoms of schizophrenia [1].

The anti-addictive potential of CBD was demonstrated in animal models of cannabis, opioid, alcohol, methamphetamine, and cocaine use disorders. Although CBD's molecular pathways are still poorly understood, they may include neuronal excitability, $5 \mathrm{HT}_{1 \mathrm{~A}}$ receptors and possibly cannabinoid and opioid systems. In small-scale clinical trials, CBD reduced cigarette consumption and heroin cue-induced craving. The anecdotal evidence also shows the positive effects of CBD on reducing symptoms of cannabis and alcohol use disorders, yet these effects need further investigation in larger trials [1].

In summary, the complexity of CBD pharmacology is due to CBD's ability to interact with several molecular targets, making it a good candidate for further therapeutic investigation. Currently, in the US, CBD is only prescribed for treatment of childhood epilepsies, while other indications are still under exploration. To fully elucidate its true therapeutic potential in other brain disorders, CBD needs to be tested in larger-scale randomized, placebo-controlled trials.

\section{FUNDING AND DISCLOSURE}

BLF has received in-kind donation of cannabis products from Canopy Innovations Inc. and Aurora Cannabis and medication donation from Pfizer and Bioprojet. He was provided a coil for the TMS study from Brainsway. BLF will conduct research with funding obtained from Canopy Innovations Inc. and Aphria (through research grants handled by $\mathrm{CAMH}$ and University of Toronto), Bioprojet, ACS and Alkermes. BLF has received in kind donations of nabiximols from GW Pharma for studies funded by $\mathrm{CIHR}$ and $\mathrm{NIH}$. The authors declare no competing interests.

\footnotetext{
${ }^{1}$ Translational Addiction Research Laboratory, Centre for Addiction and Mental Health (CAMH), 33 Russell Street, T-700, Toronto, ON M5S 2S1, Canada; ${ }^{2}$ The Institute of Medical

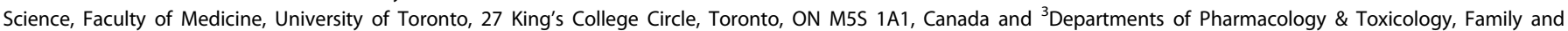
Community Medicine and Psychiatry, University of Toronto, 27 King's College Circle, Toronto, ON M5S3H7, Canada

Correspondence: Bernard Le Foll (Bernard.lefoll@camh.ca)
}

Published online: 11 September 2019 